\title{
Survival Outcomes of Twin-Twin Transfusion Syndrome Stage I: A Systematic Review of Literature
}

\author{
A. Cristina Rossi, MD ${ }^{1}$ Vincenzo D’Addario, MD ${ }^{1}$ \\ ${ }^{1}$ Clinic of Obstetrics and Gynecology, University of Bari, Bari, Italy \\ Am J Perinatol 2013;30:5-10.
}

Address for correspondence and reprint requests A. Cristina Rossi, MD, Via Celentano, 42, Bari 70121, Italy (e-mail: acristinarossi@yahoo.it).

Abstract


Keywords
- twin-twin transfusion
syndrome (TTTS)
- Quintero staging
system
- stage I
- laser therapy
- conservative
management

Objective To review literature about treatment of twin-twin transfusion syndrome (TTTS) stage I.

Materials A search in PubMed, EMBASE, Medline, and reference lists was performed. Inclusion criteria were TTTS diagnosed with standard criteria and classified with Quintero staging and survival rates (SR) stratified for stage and first-choice treatment. Outcomes were SR and progression to advanced stages. Preferred Reporting Items for Systematic reviews and Meta-Analyses guidelines were followed.

Results Seven articles pooled 262 twin sets treated with amnioreduction (16\%), conservative management (22\%), and laser therapy (62\%). The overall SR was $77 \%$ after amnioreduction, $86 \%$ in the conservative management group, and $85 \%$ in the laser therapy group. Progression rate occurred in $30 \%, 15 \%$, and $0 \%$, respectively. Outcomes were similar between laser therapy as first- versus second-choice treatment. Because most studies were observational and not comparative, a meta-analysis was not performed.

Conclusion Conservative management of TTTS stage I is a reasonable option until randomized clinical trials are presented.
Twin-twin transfusion syndrome (TTTS) complicates 10 to $15 \%$ of monochorionic twin pregnancies. ${ }^{1}$ It is well known that TTTS occurs because of unequal blood exchange from one twin (the donor) to the co-twin (the recipient) through placental vascular anastomoses. Once the diagnosis is posed, severity of TTTS is generally classified according to Quintero staging system based on sonographic findings. ${ }^{2}$ Although there is wide evidence that laser therapy is the optimal treatment for the advanced forms of $\mathrm{TTTS}^{3}$ treatment for stage I is actually under debate. This controversy is due to the unclear natural history of stage I. Some cases progress to most severe forms, for which laser therapy has been advocated ${ }^{4}$; other cases remain stable and might be treated with a less invasive procedure, such as amniocentesis ${ }^{5}$; other cases regress and might benefit of conservative management. Laser therapy and amnioreduction are invasive procedures and are occasionally complicated with iatrogenic rupture of membranes and infections, whereas conservative management is safer but the efficacy has not been definitively proven.

Because stage I occurs in a minority of TTTS cases, studies are limited by the small sample size, making it hard to derive conclusion about the optimal management of TTTS stage I. We therefore performed a systematic review of survival rates in stage I to get a large sample size and define what treatment is associated with better outcomes according to literature.

\section{Materials and Methods}

A search in PubMed, Medline, EMBASE, reference lists, and Clinical Trials Registry was performed. Because Quintero received

February 10, 2012

accepted after revision

March 15, 2012

published online

July 26, 2012
Copyright $\odot 2013$ by Thieme Medical Publishers, Inc., 333 Seventh Avenue, New York, NY 10001, USA. Tel: +1(212) 584-4662.
DOI http://dx.doi.org/ 10.1055/s-0032-1322513. ISSN $0735-1631$. 
staging system was introduced in 1999, the search was limited from 1999 through 2011. Key words were: twintwin transfusion syndrome (TTTS), laser therapy, Quintero stage (staging), stage I, (serial) amnioreduction, conservative management, expectant management. Studies were selected according to the following inclusion criteria: TTTS diagnosed according to standard criteria and classified according to Quintero staging system, survival rates of twins in stage I reported as proportional rates, TTTS diagnosed in the second trimester. Exclusion criteria were omitting at least one inclusion criteria, TTTS treated with selective feticide, survival rates not stratified for stages, TTTS diagnosed in the third trimester, data reported in graphs or percentage, non-English language publications, and personal communications.

Standard criteria for diagnosis of TTTS consisted in oligohydramnios in the donor's sac (maximal vertical pocket $\leq 2 \mathrm{~cm}$ ) and polyhydramnios in the recipient's sac (maximal vertical pocket $\geq 8 \mathrm{~cm}$ ). Quintero staging system defined severity of TTTS according to sonographic criteria as follow: stage I: visualization of donor's bladder; stage II: bladder not visualized in the donor twin; stage III: abnormal Doppler of the umbilical artery and/or ductus venosus in one or both twins; stage IV: hydrops in one or both twins; stage V: intrauterine demise of one or both twins. ${ }^{2}$

First-choice treatment, survival rates of stage I, and progression to stages II to $\mathrm{V}$ were abstracted from each article. Survival rates were calculated for each twin set (no survivors, one survivor, two survivors, at least one survivor) and overall. When data were missing, an attempt to contact the corresponding author was performed to obtain unpublished outcomes.

Survival outcomes were classified in three groups according to the first-choice treatment (i.e., amnioreduction, conservative management, and laser therapy).

The systematic review was performed according to the Preferred Reporting Items for Systematic reviews and MetaAnalyses guidelines. Study selection bias was assessed according to the Cochrane guidelines. The two authors independently selected articles. Discordance was resolved with consensus.

\section{Results}

-Figure 1 shows the steps of the review. Seven articles reported neonatal survival rates of stage I. ${ }^{4,6-11}$ Characteristics of studies included in the review are reported in - Table 1. Overall, there were 262 twin sets, of which first-choice treatment was amnioreduction in 43 (16\%), conservative management in 59 (22\%), and laser therapy in 160 (62\%).

In the amnioreduction group, the overall survival rate was 66/86 (77\%) twins. There were no survivors in 3/43 (7\%) twin sets, one survivor in 14/43 (33\%) twin sets, and two survivors in 26/43 (60\%) twin sets. At least one twin survived in $40 / 43$ (93\%). Progression of stage I toward more severe stages was observed in 13/43 (30\%) cases. Outcomes of these progressed cases were reported in four twin sets, of which second-choice treatment was laser

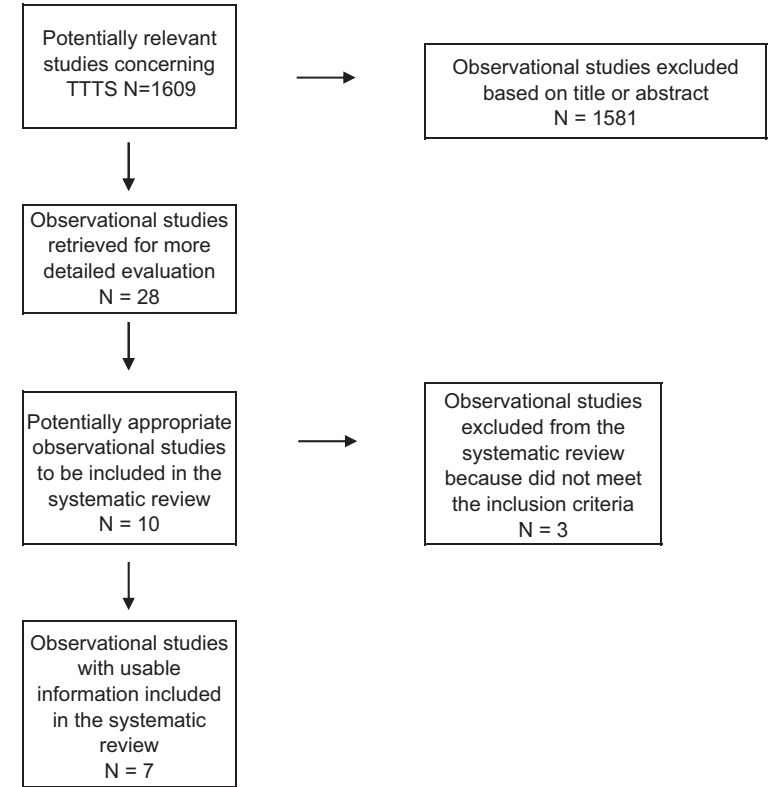

Figure 1 Steps of study selection. TTTS, twin-twin transfusion syndrome.

therapy in two cases, bipolar cord coagulation in one case, and amnioreduction in one case.

In the conservative management group, the overall survival rate accounted for 102/118 (86\%) twins. No survivors were reported in 3/59 (5\%) twin sets, one survivor was observed in 10/59 (17\%) twin sets, and two survivors were noted in 46/59 (78\%) twin sets. At least one survivor was reported in 56/59 (95\%) twin sets. Progression of stage I toward advanced stages occurred in 6/39 (15\%) cases (20 cases unreported). All the progressed cases were treated by laser therapy. Therefore, laser therapy was performed as second-choice treatment in eight cases (two after amnioreduction and six after conservative management).

In the laser therapy group, the overall survival rate was $272 / 320$ (85\%) twins. There were no survivors in $12 / 160$ (7\%) twin sets, one survivor in 24/160 (15\%) twin sets, and two survivors in $124 / 160$ (78\%) twin sets. At least one survivor was noted in 148/160 (93\%) twin sets. No progression was reported in fetuses treated with laser therapy. Survival rates of laser therapy performed as first or second choice are reported in -Fig. 2.

-Figure 3 summarizes outcomes according to first-choice treatment.

Only one article compared outcomes following laser therapy versus conservative management and did not find statistically significant differences in survival rates. ${ }^{4}$

The included studies were heterogeneous: one article described outcomes following amnioreduction, ${ }^{8}$ one article compared amnioreduction with conservative management, ${ }^{6}$ three articles used laser therapy as first-choice treatment, ${ }^{7,9,11}$ one article compared laser therapy with conservative management, ${ }^{4}$ and one article focused on conservative management. ${ }^{10}$ Due to this heterogeneity (and because all but two studies were observational and not comparative), a 


\begin{tabular}{|c|c|c|c|c|c|c|c|c|c|}
\hline 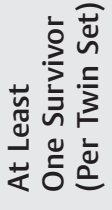 & \begin{tabular}{|l} 
\\
$\stackrel{\circ}{\circ}$ \\
$\tilde{m}$ \\
$\alpha$ \\
$\grave{N}$
\end{tabular} & $\begin{array}{l}\widehat{ㅇ} \\
\stackrel{\circ}{\circ} \\
= \\
-\end{array}$ & $\begin{array}{l}\text { ஓo } \\
\text { すे } \\
\text { m. } \\
\text { Oे }\end{array}$ & 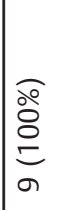 & 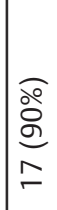 & $\begin{array}{l}\bar{o} \\
\bar{\vdots} \\
\hat{a} \\
\text { Na }\end{array}$ & 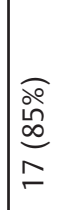 & 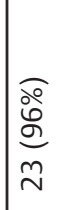 & 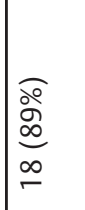 \\
\hline 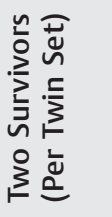 & 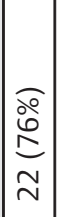 & 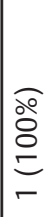 & $\begin{array}{l}\widehat{\widehat{\rho}} \\
\stackrel{\infty}{0} \\
\infty \\
\infty\end{array}$ & 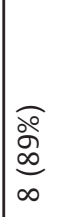 & 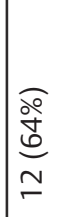 & $\begin{array}{l}\bar{o} \\
\stackrel{\circ}{\hat{N}} \\
\text { N }\end{array}$ & $\begin{array}{l}\text { 웅 } \\
\hat{\mathrm{h}} \\
\text { m }\end{array}$ & 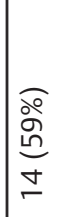 & 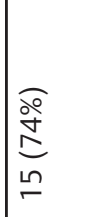 \\
\hline 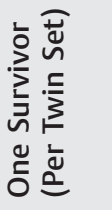 & $\begin{array}{l}0 \\
\stackrel{\circ}{N} \\
\\
\text { nn }\end{array}$ & 六 & 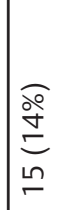 & 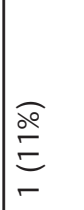 & $\begin{array}{l}\widehat{\stackrel{\circ}{\circ}} \\
\stackrel{\varrho}{\mathrm{d}} \\
\text { Ln }\end{array}$ & 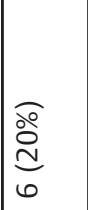 & 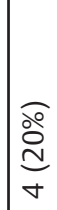 & $\begin{array}{l}\widehat{\curvearrowright} \\
\stackrel{\circ}{0} \\
\sigma\end{array}$ & 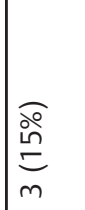 \\
\hline 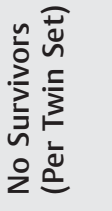 & 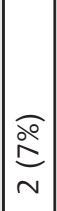 & 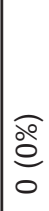 & 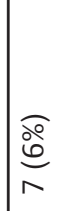 & 仓̊ & $\begin{array}{l}\stackrel{0}{\circ} \\
\stackrel{\circ}{\complement} \\
\sim\end{array}$ & 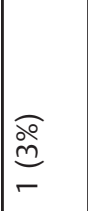 & 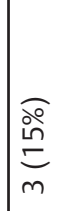 & 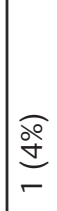 & $\frac{\widehat{\partial}}{\stackrel{\partial}{c}}$ \\
\hline 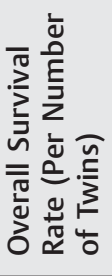 & 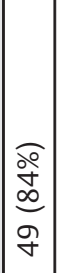 & 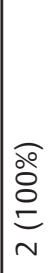 & 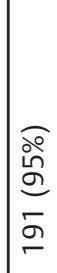 & 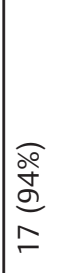 & 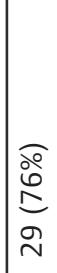 & 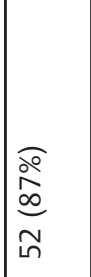 & 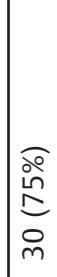 & 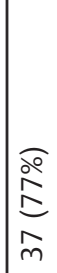 & $\begin{array}{l}\text { } \\
\grave{o} \\
\infty \\
m \\
m\end{array}$ \\
\hline 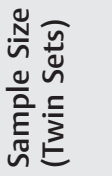 & $\stackrel{N}{ }$ & - & 음 & $a$ & a & 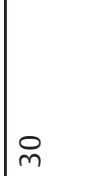 & i & $\stackrel{\beth}{\sim}$ & $\stackrel{i}{\text { N }}$ \\
\hline 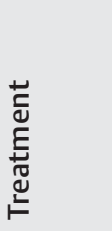 & 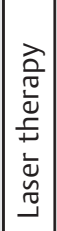 & 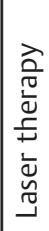 & 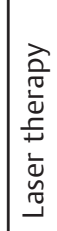 & 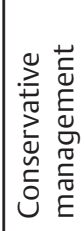 & 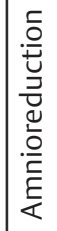 & 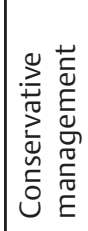 & 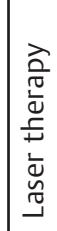 & 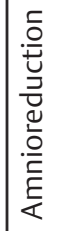 & 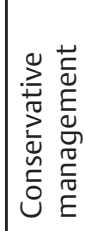 \\
\hline 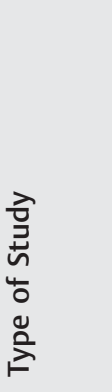 & 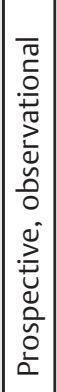 & 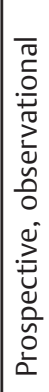 & 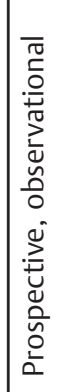 & 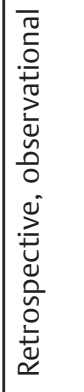 & 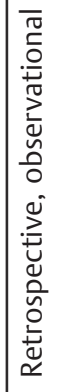 & 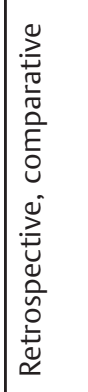 & & 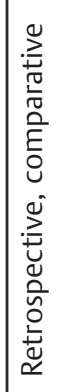 & \\
\hline 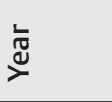 & $\begin{array}{l}0 \\
\stackrel{\vdots}{0} \\
\text { | }\end{array}$ & $\frac{\circ}{\stackrel{0}{N}}$ & $\bar{\nu}$ & $\frac{O}{\grave{N}}$ & $\frac{0}{\tilde{N}}$ & ஓे̀ & & $\frac{0}{\sim}$ & \\
\hline 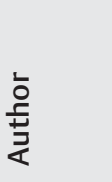 & 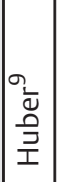 & 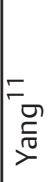 & & 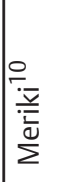 & 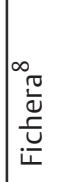 & 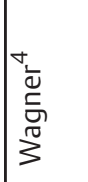 & & 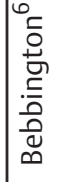 & \\
\hline
\end{tabular}

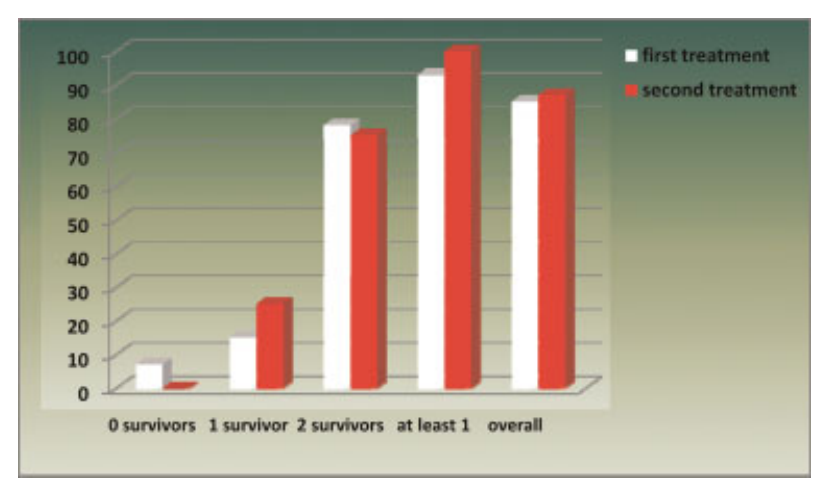

Figure 2 Survival rates of laser therapy as first- and second-choice treatment.

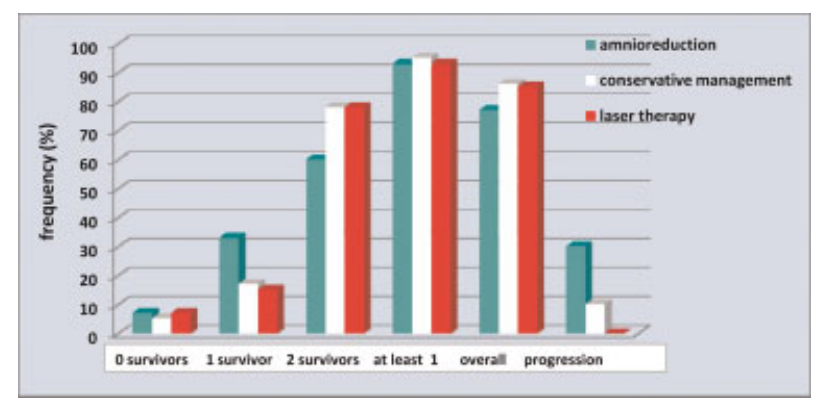

Figure 3 Survival rates according to first-choice treatment.

meta-analysis could not be performed because it was not possible to compare a specific study group with a control group.

\section{Discussion}

This review shows that amnioreduction for TTTS in stage I is associated with a low overall survival rate. Because of the wide evidence that amnioreduction results in adverse neonatal outcomes even in the mildest form of TTTS, as demonstrated by our review, amnioreduction as treatment for TTTS should be definitively abandoned. In contrast, conservative management represents a safe option for TTTS stage I because survival rates are not clinically different from those obtained following laser therapy ( $86 \%$ and $85 \%$, respectively).

Our review disagrees with the old paradigm according to which "TTTS is associated with $80-100 \%$ mortality, if left untreated." 12 In fact, we found that in the conservative management group, mortality rate accounts for $14 \%$ (overall survival rate: $86 \%$ ) and only $13 \%$ of untreated cases worsen toward advanced stages. It is generally believed that laser therapy is the optimal treatment for TTTS, because it interrupts intertwin blood exchange by photocoagulation of placental anastomoses. According to our results, this statement does not apply for the mildest form of TTTS, which can be managed conservatively in the majority of cases. This might be due to the higher number of the protective arteroarterial anastomoses that can be observed in stage I ending with regression or stable disease compared with stage I 
progressing to stage II to $\mathrm{V}$. Another explanation for which the survival rates of stage I following conservative management are as good as following laser therapy might be that stage I does not describe severity of TTTS appropriately. Stage I should be the first step to define the severity of TTTS, but "visualization of donor's bladder" is a normal sonographic finding that is observed in all healthy fetuses. Therefore, the definition of stage I does not mark a pathological sign and does not provide additional information about the first step of TTTS, but simply overlaps with the definition of TTTS. Another limitation is that stage I (together with stage II, i.e., no visualization of donor's bladder) does not include the examination of the recipient. Therefore, it is not possible to assess recipient twins in the early forms of TTTS. The use of a single staging system to assess severity in two twins, which differ with regard to symptomatology, might generate confusion. In particular, twin-twin "confusion" syndrome is generated if we try to classify severity of TTTS in those cases presenting the recipient twin with hydrops (stage IV) and the donor cotwin with visible bladder (stage I). In this paradoxical case, the recipient would benefit from laser therapy (i.e., the most invasive procedure for TTTS), whereas the donor might be managed conservatively (i.e., the less invasive procedure). We recently proposed a double staging system, namely "Rossi staging system," to quantify the severity of TTTS in the two twins independently. ${ }^{13}$

From our review, the progression rate is clinically higher in the amnioreduction (30\%) than conservative management (15\%) and laser therapy (0\%) groups. Although conservative management is associated with an increased risk of progression, the overall survival rate of laser therapy as secondchoice treatment (87\%) is clinically similar to those of laser therapy as first-choice treatment (85\%). Therefore, in the subgroup of twins undergoing conservative management followed by laser therapy if the syndrome progressed, the delay in performing laser therapy does not represent a risk factor for adverse outcomes.

Caution should be exercised in interpreting our results. One limitation of our review is represented by the lack of statistical power to compare different procedures because all but two studies ${ }^{4,6}$ were observational and not comparative. Therefore, results must be interpreted clinically. From a clinical point of view, we believe that the overall survival rates are similar between conservative management and laser therapy (86\% versus $85 \%$, respectively) but are clinically different compared with the overall survival rate in the amnioreduction group (77\%). Twins treated with amnioreduction as first-choice treatment also present a clinically relevant risk of progression (30\%) compared with twins managed expectantly (15\%) or treated with laser therapy $(0 \%)$.

Another limitation is that survival rates in twins affected with TTTS are influenced by multiple variables, such as gestational age at diagnosis and treatment, gestational age at delivery, birth weight discordance, placental territory, and type of intertwin anastomoses. However, the reviewed articles did not stratify neonatal survival for these perinatal characteristics. Only one article assessed neonatal morbidity and found adverse short- and long-term neurodevelopmental outcomes significantly worse in the conservative management than laser therapy group. ${ }^{4}$

The reviewed articles did not describe placental characteristics. Although the study sample presented the same level of severity (i.e., stage I), it is likely that different patterns of placental anastomoses might have contributed to bias selection.

Finally, survival rates were not classified according to type of twin. Because stage I is defined by assessing only the donor twin, in our opinion it would be important to compare donors' with recipients' survival.

Despite these limitations, our review has the strength of a large sample size, which is essential when clinical conditions are infrequent, as the case of TTTS stage I.

In conclusion, literature shows that amnioreduction is not an efficacious treatment of TTTS stage I. Although a statistical analysis cannot be performed with available data, survival findings were not clinically different between conservative management and laser therapy. Topics for future research that might be helpful to understand the natural history of stage I TTTS should focus on a better definition of stage I and probably the whole staging system, placental characteristics, factors that might predict stage I outcomes, and randomized clinical trials comparing conservative management with laser therapy. Until randomized clinical trials become available, conservative management should be considered a reasonable option.

\section{Note}

This review was presented by the first author to the First World Conference in Pediatrics and Obstetrics/Gynecology, OMICS group, Philadelphia, Pennsylvania (December 6 to 8 , 2011) as oral presentation.

\section{References}

1 Berghella V, Kaufmann M. Natural history of twin-twin transfusion syndrome. J Reprod Med 2001;46:480-484

2 Quintero RA, Morales WJ, Allen MH, Bornick PW, Johnson PK, Kruger M. Staging of twin-twin transfusion syndrome. J Perinatol 1999;19(8 Pt 1):550-555

3 Rossi AC, D'Addario V. Laser therapy and serial amnioreduction as treatment for twin-twin transfusion syndrome: a metaanalysis and review of literature. Am J Obstet Gynecol 2008;198:147-152

4 Wagner MM, Lopriore E, Klumper FJ, Oepkes D, Vandenbussche FP, Middeldorp JM. Short- and long-term outcome in stage 1 twin-totwin transfusion syndrome treated with laser surgery compared with conservative management. Am J Obstet Gynecol 2009;201: 286, e1-e6

5 O'Donoghue K, Cartwright E, Galea P, Fisk NM. Stage I twin-twin transfusion syndrome: rates of progression and regression in relation to outcome. Ultrasound Obstet Gynecol 2007;30:958-964

6 Bebbington MW, Tiblad E, Huesler-Charles M, Wilson RD, Mann SE, Johnson MP. Outcomes in a cohort of patients with stage I twinto-twin transfusion syndrome. Ultrasound Obstet Gynecol 2010; 36:48-51

7 Chmait RH, Kontopoulos EV, Korst LM, Llanes A, Petisco I, Quintero RA. Stage-based outcomes of 682 consecutive cases of twin-twin transfusion syndrome treated with laser surgery: the USFetus experience. Am J Obstet Gynecol 2011;204:393, e1-e6 
8 Fichera A, Lanna M, Fratelli N, Rustico M, Frusca T. Twin-to-twin transfusion syndrome presenting at early stages: is there still a possible role for amnioreduction? Prenat Diagn 2010;30:144-148

9 Huber A, Diehl W, Bregenzer T, Hackelöer BJ, Hecher K. Stagerelated outcome in twin-twin transfusion syndrome treated by fetoscopic laser coagulation. Obstet Gynecol 2006;108:333-337

10 Meriki N, Smoleniec J, Challis D, Welsh AW. Immediate outcome of twin-twin transfusion syndrome following selective laser photocoagulation of communicating vessels at the NSW Fetal Therapy Centre. Aust N Z J Obstet Gynaecol 2010;50:112-119
11 Yang X, Leung TY, Ngan Kee WD, Chen M, Chan LW, Lau TK. Fetoscopic laser photocoagulation in the management of twintwin transfusion syndrome: local experience from Hong Kong. Hong Kong Med J 2010;16:275-281

12 Jain V, Fisk NM. The twin-twin transfusion syndrome. Clin Obstet Gynecol 2004;47:181-202

13 Rossi AC, D'Addario V. The efficacy of Quintero staging system to assess severity of twin-twin transfusion syndrome treated with laser therapy: a systematic review with meta-analysis. Am J Perinatol 2009;26:537-544 